\title{
Amplitude and Phase of the Low-Frequency Ground Wave Near a Coastline
}

\author{
James R. Wait
}

\begin{abstract}
A theoretical analysis is given for the amplitude and the phase change of the ground wave, originating from a distant transmitter on land, as it crosses a coastline. The land and sea are assumed to be smooth, and homogeneous with a sharp boundary of separation. Attention is focused on the effects that take place near the coastline when it is not permissible to employ arguments based on the principle of stationary phase. A limited comparison is made with the recent experimental work of Pressey, Ashwell, and Fowler.
\end{abstract}

\section{Introduction}

With the increased use of low-frequency radio navigational systems, it has become important to understand the mechanism of ground-wave propagation over inhomogeneously conducting terrain. Theoretical treatments of this problem usually employ an idealized twopart media having a sharp boundary separating homogeneous media. The extension of these analyses to three or more media is straightforward, although tedious. In most of the previous work, ${ }^{1}$ the numerical values of the fields are only valid for distances from the boundary greater than a wavelength or so, as a consequence of the approximations employed. It is the purpose of the present note to investigate the field near the boundary by a refinement of the stationary phase method.

\section{Theoretical Development}

In an earlier paper (see footnote 1), analysis and computations were presented for the propagation of ground waves over a mixed path on a flat earth. The boundary separating the two media of contrasting conductivity was assumed to be straight and approximately normal to the propagation path. A relation was derived for the mutual impedance $Z^{\prime}$ between the terminals of the transmitting and the receiving antennas, both of which are represented by dipoles. The conductivity of the earth to the left of the boundary is denoted by $\sigma$ and that to the right by $\sigma_{1}$. It is convenient to let

$$
Z^{\prime}=Z+\Delta Z
$$

where $Z$ is the mutual impedance for the two antennas, $\mathrm{A}$ and $\mathrm{B}$, corresponding to an earth that is all homogeneous with conductivity $\sigma$ and $\Delta Z$ is the increment to account for the portion of the path to the right of the boundary with conductivity $\sigma_{1}$. From the early work it follows that

$$
Z=-\frac{h_{a} h_{b} i \mu \omega}{2 \pi\left(r_{0}+R_{0}\right)} e^{-i \beta\left(r_{0}+R_{0}\right)} F\left(r_{0}+R_{0}, \eta\right)\left[1+\frac{1}{i \beta\left(r_{0}+R_{0}\right)}-\frac{1}{\beta^{2}\left(r_{0}+R_{0}\right)^{2}}\right],
$$

where $\beta=2 \pi /$ free-space wavelength, and $h_{a}$ and $h_{b}$ are the effective heights of the antennas at $\mathrm{A}$ and $\mathrm{B}, \mu=4 \pi \times 10^{-7}, \omega=$ angular frequency, $\eta=(i \mu \omega / \sigma)^{1 / 2} . \quad F\left(r_{0}+R_{0}, \eta\right)$ is a slowly varying Sommerfeld attenuation function defined previously (see footnote 1 ), and is a function of the separation distance $r_{0}+R_{0}$ and surface impedance $\eta$.

1 J. R. Wait, Mixed path ground wave propagation; 1 . Short distances, J. Research NBS 57, 1 (1956) RP2687. References to the work of Millington, Clemmow, Bremmer, Furutsu, and others are given here. 


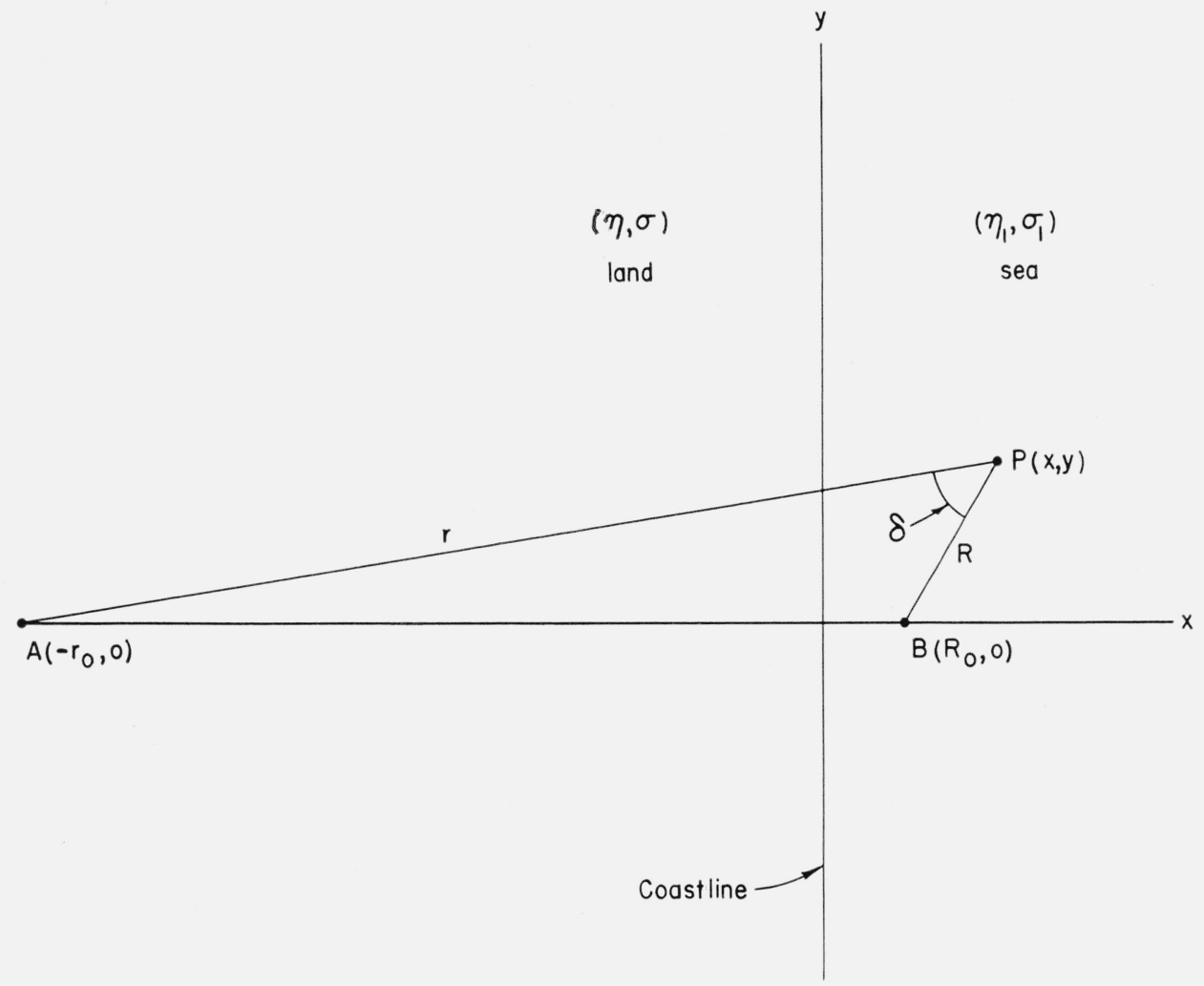

FIGURE 1. Schematic representation of a land-sea boundary with a transmitter at A and a receiver at B.

The corresponding expression for the increment $\Delta Z$ is an integration over the media to the right of the boundary, which, in figure 1, corresponds to positive values of $x$;

$$
\Delta Z=\frac{\left(\eta_{1}-\eta\right) \beta^{2} h_{a} h_{b}}{4 \pi^{2}} \int_{x=0}^{\infty} \int_{y=-\infty}^{+\infty} \frac{e^{-\beta(r+R)}}{r R} F(r, \eta) F^{\prime}\left(R, \eta, \eta_{1}\right)\left(1+\frac{1}{i \beta R}\right)\left(1+\frac{1}{i \beta r}\right) \cos \delta d x d y,
$$

where $r$ and $R$ are the distances from the variable point $\mathrm{P}$ to $\mathrm{A}$ and $\mathrm{B}$, respectively, and $\delta$ is the angle between unit vectors at $\mathrm{P}$ in the direction of increasing $r$ and $R$. The attenuation function $F(r, \eta)$ is of the same form as that in eq $(2)$; however, $F^{\prime}\left(R, \eta, \eta_{1}\right)$ is some unknown slowly varying function that depends on $R$, $\eta$, and $\eta_{1}\left[=\left(i \mu \omega / \sigma_{1}\right)^{1 / 2}\right]$. By utilizing a stationary phase principle, the area integration can be reduced to a line integral from $x=0$ to $R_{0}$, enabling a solution to be carried out. To this approximation, $F^{\prime}\left(R, \eta, \eta_{1}\right)$ could be replaced by $F\left(R, \eta_{1}\right)$. It was admitted that this procedure leads to a result that is not valid near the boundary. As a further consequence of this stationary phase argument, the reflected wave from the boundary is neglected. In the present instance, $R_{0}$ is considered small compared with $r_{0}$. It then can be anticipated that the important contribution of the integration will be confined to a region where $x$ and $y$ are small compared with $r_{0}$. In this region it is permissible to assume:

$$
\begin{aligned}
F(r, \eta) & \cong F\left(r_{0}, \eta\right) \cong F\left(r_{0}+R_{0}, \eta\right), \\
F^{\prime}\left(R, \eta, \eta_{1}\right) & \simeq 1, \quad\left(\text { subject to } \sigma_{1}>>\sigma\right) \\
\cos \delta & \cong\left(x-R_{0}\right) / R, \quad \beta(r+R) \cong \beta\left(r_{0}+x+R\right) .
\end{aligned}
$$

In other words, the boundary is in the Fraunhofer field of the source at A. Then it follows that

$$
\Delta Z \cong\left(\eta_{1}-\eta\right) \frac{\beta^{2} h_{a} h_{b}}{4 \pi^{2} r_{0}} e^{-i \beta r_{0}} \boldsymbol{F}\left(r_{0}, \eta\right)
$$




$$
\int_{x=0}^{\infty} e^{-i \beta x} \int_{y=-\infty}^{+\infty} \frac{e^{-i \beta R}}{R} \frac{\left(x-R_{0}\right)}{R}\left(1+\frac{1}{i \beta r}\right) d y d x
$$

where

$$
R \cong\left[\left(x-R_{0}\right)^{2}+y^{2}\right]^{1 / 2} .
$$

It is now noted that

$$
\int_{0}^{\infty} \frac{e^{-i \beta R}}{R} d y=-\frac{\pi i}{2} H_{0}^{(2)}\left[\beta\left|x-R_{0}\right|\right]
$$

which is a consequence of Campbell and Fosters ${ }^{2}$ pair No. 917, which requires $\beta$ to have a finite negative imaginary part. $H_{0}^{(2)}$ is the Hankel function of the second kind. Using this result, it follows that

$$
\Delta Z=\frac{\left(\eta_{1}-\eta\right) i \beta h_{a} h_{b}}{2 \pi^{2} r_{0}} e^{-i \beta r_{0} F\left(r_{0}, \eta\right) I}
$$

where

$$
I=-\frac{\pi i}{2} \int_{x=0}^{\infty} e^{-i \beta x} \frac{d}{d x} H_{0}^{(2)}\left[\beta\left|x-R_{0}\right|\right] d x .
$$

Integrating by parts and changing the integration variable leads to

$$
I=\frac{\pi i}{2}\left\{H_{0}^{(2)}\left(\beta R_{0}\right)-i e^{-i \beta R}\left[\int_{0}^{\beta R_{0}} H_{0}^{(2)}(z) e^{+i z} d z+\int_{0}^{\infty-i \epsilon} H_{0}^{(2)}(z) e^{-i z} d z\right]\right\}
$$

for $R_{0}>0$, and

$$
I=\frac{\pi i}{2}\left\{H_{0}^{(2)}\left(-\beta R_{0}\right)-i e^{-i \beta R_{0}} \int_{-\beta R_{0}}^{\infty-i \epsilon} H_{0}^{(2)}(z) e^{-i z} d z\right\}
$$

for $R_{0}<0$, where $\epsilon$ is any arbitrarily small real number. ${ }^{3}$

Now it can be readily verified that

$$
\frac{\partial}{\partial z}\left\{z e^{ \pm i z}\left[H_{0}^{(2)}(z) \mp i H_{1}^{(2)}(z)\right]\right\}=e^{ \pm i z} H_{0}^{(2)}(z)
$$

so the integrations can be carried to yield

$$
I=i \frac{\pi}{2}\left[\left(1-i \beta R_{0}\right) H_{0}^{(2)}\left(\beta R_{0}\right)-\beta R_{0} H_{1}^{(2)}\left(\beta R_{0}\right)\right]
$$

for $R_{0}>0$, and

$$
\left.I=i \frac{\pi}{2}\left(1-i \beta R_{0}\right) H_{0}^{2)}\left(-\beta R_{0}\right)+\beta R_{0} H_{1}^{(2)}\left(-\beta R_{0}\right)\right]
$$

for $R_{0}<0$. After a little substitution and cancellation, it is easy to show that

$$
\begin{aligned}
\frac{Z^{\prime}}{Z} & =1+\frac{\Delta Z}{Z} \\
& =1+q e^{\frac{3 i \pi}{4}} e^{i \alpha}\left[(1-i \alpha) H_{0}^{(2)}(\alpha)-\alpha H_{1}^{(2)}(\alpha)\right]
\end{aligned}
$$

${ }^{2}$ A. N. Campbell and R. Foster, Fourier integrals (D. Van Nostrand Co., Inc., New York, N. Y., 1948).

${ }_{3}$ The finite value of $\epsilon$ insures that the integrals converge in the Riemann sense. After'carrying out the evaluation, $\epsilon$ can be set equal to zero. 
with $\alpha=\beta R_{0}>0$, and

$$
\frac{Z^{:}}{Z}=1+q e^{\frac{3 i \pi}{4}} e^{-i \alpha}\left[(1+i \alpha) H_{0}^{(2)}(\alpha)-\alpha H_{1}^{(2)}(\alpha)\right]
$$

with $\alpha=\beta R_{0}>0$. In the above

$$
q=\frac{\eta-\eta_{1}}{\eta}\left|\frac{\eta}{\eta_{0}}\right| \cong 0.0075\left(1-\sqrt{\frac{\sigma}{\sigma_{1}}}\right)\left(f_{m c} / \sigma\right)^{1 / 2},
$$

where $f_{m c}$ is the frequency in megacycles per second; $q$ is a measure of the conductivity contrast between the two media and is a positive or negative real number if displacement currents in the ground can be neglected. Also, as a consequence of the approximate boundary conditions, $|\eta|<<\eta_{0}=120 \pi$, and therefore $|\Delta Z| Z \mid$ is restricted to values small compared with unity.

\section{Discussion of Results}

It is instructive, at this stage, to discuss the behavior of the ratio $Z^{\prime} / Z$ for large $\alpha$ (i. e., large electrical distances from the boundary). The asymptotic developments for the Hankel function can then be employed, to yield

when $\mathrm{R}_{0}>0$, and

$$
1+\frac{\Delta Z}{Z} \cong 1+i q\left(\frac{2 \alpha}{\pi}\right)^{1 / 2}\left[1+i \frac{3}{8 \alpha}-\frac{5}{128 \alpha^{2}}+\ldots\right]
$$

$$
1+\frac{\Delta Z}{Z} \cong 1-q\left(\frac{1}{2 \pi \alpha}\right)^{1 / 2} e^{-2 i \alpha}\left[1-\frac{i}{24 \alpha}+\ldots\right]
$$

when $\mathrm{R}_{0}<0$. It is most significant to note that the right-hand side of eq (17), when the squarebracket term is replaced by unity, corresponds to the first term of a development in higher powers of $\sqrt{\alpha}\left(\eta_{1} / \eta_{0}\right)$ obtainable from the earlier paper (see appendix). That is, the formulas derived by the stationary phase methods are probably only valid if $\alpha \gg 1$ corresponding to $\mathrm{R}_{0}$ large compared to the wavelength. On the other hand, the stationary phase development would yield a value of unity for the right-hand side of eq (18). This would also be valid at large distances, in wavelengths, from the boundary. The departure from unity can be interpreted as a reflected wave from the boundary that forms, with the incident wave, a standing wave pattern on the transmitter side of the boundary. A more detailed view of the situation is illustrated in figures 2 and 3 , where the amplitude and phase of $(1+\Delta Z / Z)$ are shown plotted as a function of $\mathrm{R}_{0} / \lambda$ for various values of $q$. It is interesting to note that very near the boundary the field has a logarithmic singularity because of the behavior of the zero-order Hankel function as its argument approaches zero. It is believed that the solution is not strictly valid for very small values of $\alpha$ because the approximate boundary condition introduced in the earlier paper is not applicable at or close to a discontinuity in the surface impedance. ${ }^{4}$

The appropriate values of the amplitude and phase $1+\Delta Z / Z$ derivable by stationary phase methods are also shown in figures 2 and 3 and indicated by dotted curves. There are significant differences between the two methods of calculations for regions within a few wavelengths of the boundary. However, the phase curves on the seaside of the boundary are almost identical to stationary-phase evaluated case, except for regions very close to the boundary.

It is interesting to compare these results with some phase measurements made very recently by Pressey, Ashwell, and Fowler. ${ }^{5} \quad$ They confirmed the presence of the phase recovery effect that is experienced by a wave passing from land to sea. They also observed systematic variations, about the median recovery effect, whose magnitudes are of the order of $5^{\circ}$ very

\footnotetext{
1 The approximate boundary conditions are not valid for $R_{0} / \lambda$ less than about $\left|\eta / \eta_{0}\right|$ or a small fraction of a wavelength for well-conducting ground.

${ }^{5}$ B. G. Pressey, G. E. Ashwell, and C. S. Fowler, Change of phase of a low frequency ground wave propagated across a coast line, Proc. Inst. Elec. Engrs. [B] 103, 527 (1956).
} 


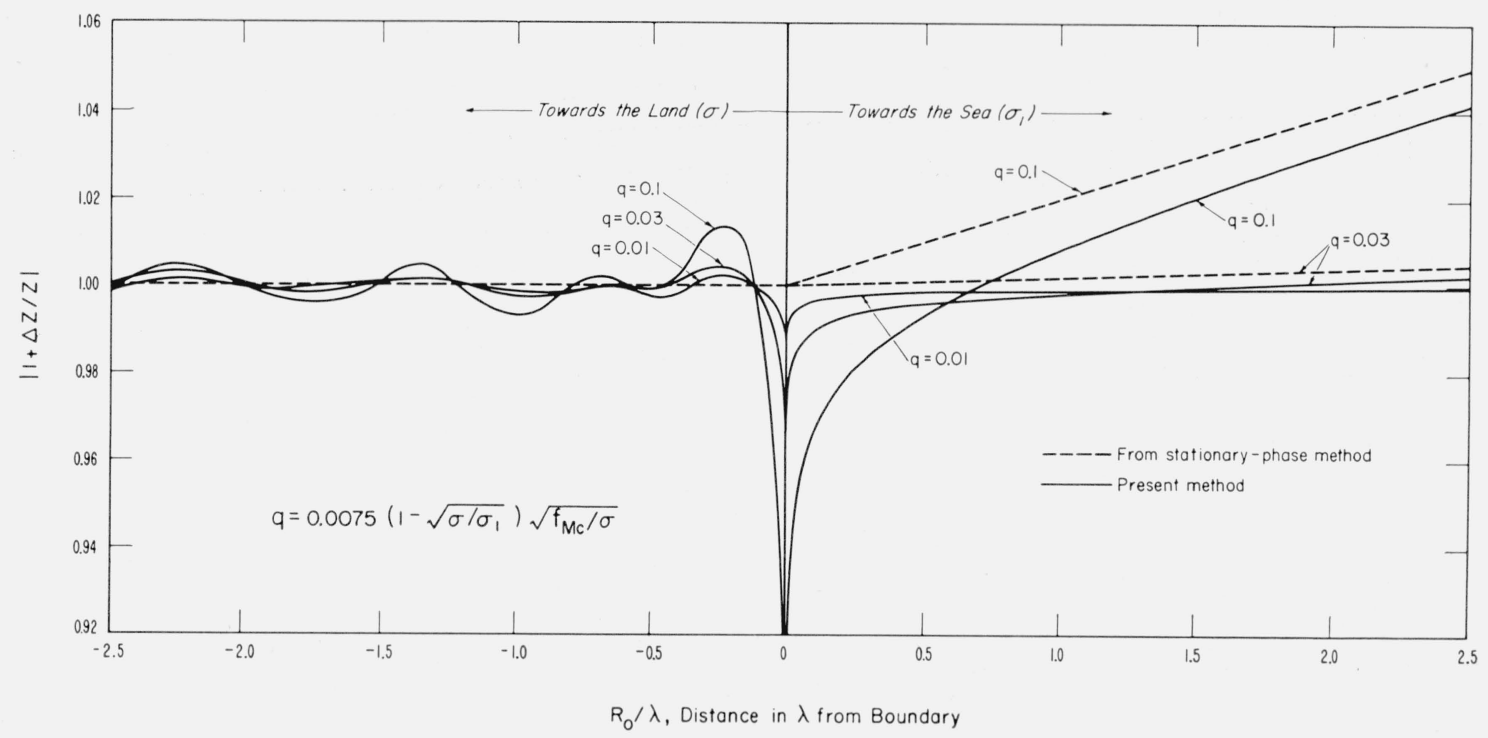

Figure 2. Relative amplitude of the field near the coastline.

The ordinate is the relative amplitude of the field at $\mathrm{B}$ for a distant transmitter at $\mathrm{A}$ on the land (fig. 1).

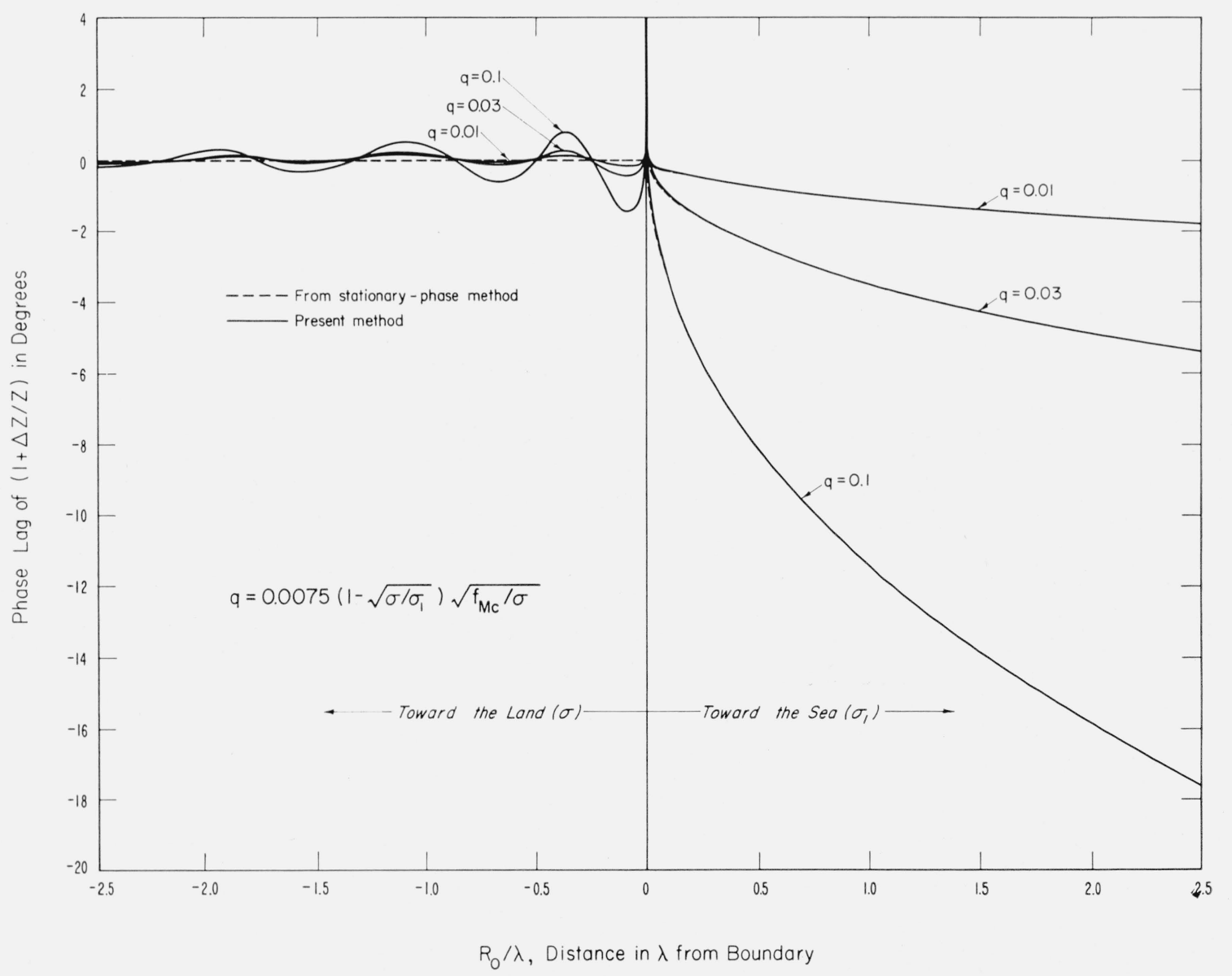

Figure 3. Relative phase of the field near the coastline.

The ordinate is the relative phase of the field at B (fig. 1). 
near the coast and decay to a negligible value within a few wavelengths from the boundary toward the sea. These variations, which are not predicted by the present theory, are of a form that varied from path to path and seemed to be dependent on the topography of the coastline. In practically all cases, however, they observed a large and rather sudden increase of phase lag very near the boundary, which is in qualitative agreement with figure 3 . There was also some evidence from Pressey, Ashwell, and Fowler's work that a standing wave does exist on the transmitter side of the boundary, although the data here is rather meager.

\section{Appendix}

The stationary phase approach gives (see footnote 1)

$$
F^{\prime}\left(r_{0}+R_{0}, \eta, \eta_{1}\right)=F\left(r_{0}+R_{0}, \eta\right)-i \epsilon \omega\left(\eta-\eta_{1}\right)\left(\frac{d}{2 \pi i \beta}\right)^{1 / 2} \int_{0}^{R_{0}} \frac{F\left(r_{0}+x, \eta\right) F\left(R_{0}-x, \eta_{1}\right)}{\left[\left(r_{0}+x\right)\left(R_{0}-x\right)\right]^{1 / 2}} d x,
$$

where

and

$$
F(x, \eta)=1-i 2 \rho^{1 / 2} \int_{i \rho^{3 / 3}}^{\infty} e^{-s^{2}} d s e^{-\rho}
$$

$$
\rho=-\frac{i \beta x}{2}\left(\frac{\eta}{\eta_{0}}\right)^{2}
$$

If $r_{0}$ is large compared to $R_{0}$, it is permissible to use the approximation

$$
\frac{F\left(r_{0}+x, \eta\right)}{\left(r_{0}+x\right)^{1 / 2}} \cong \frac{F\left(r_{0}\right)}{r_{0}^{1 / 2}}
$$

and the power series expansion of the function $F\left(R_{0}-x, \eta_{1}\right)$ to yield

where

$$
\frac{F^{\prime}}{F} \cong \frac{Z^{\prime}}{Z} \cong 1-\frac{2 i\left(1-\sqrt{\frac{\sigma_{1}}{\sigma}}\right)}{\sqrt{\pi}}\left[\rho_{1 \downarrow}^{1 / 2}-\frac{i \sqrt{\pi} \rho_{1}}{2}-\frac{2 \rho_{1}^{3 / 2}}{3}+\cdots\right],
$$

$$
\rho_{1}=-\frac{i \beta R_{0}}{2}\left(\frac{\eta_{1}}{\eta_{0}}\right)^{2}=\frac{\alpha}{2}\left|\frac{\eta_{1}}{\eta_{0}}\right|^{2}
$$

Boulder, Colo., October 25, 1956. 